\title{
INFLUÊNCIA DOS HORMÔNIOS SEXUAIS NA REGULAÇÃO FISIOLÓGICA DA RESPIRAÇÃO: UMA REVISÃO DE LITERATURA
}

\author{
Weslley Barbosa Sales \\ Renata Ramos Tomaz"
}

\begin{abstract}
RESUMO
O Sistema Respiratório é responsável por oxigenar todo o sangue que esteja pobre em oxigênio $\left(\mathrm{O}_{2}\right)$ e remover do nosso sangue o gás carbônico ( $\left.\mathrm{CO}_{2}\right)$. Mesmo que não haja regulação dos hormônios sexuais, referentes ao processo de respiração, a literatura mostra que há influência, principalmente quando estes podem apresentar ação estimulante ou inibitória sobre o sistema respiratório. O estudo teve como objetivo revisar a literatura sobre os efeitos dos principais hormônios sexuais, envolvidos na regulação fisiológica da respiração, assim como sua influência na fisiopatologia de desordens respiratórias, evidenciando sua contribuição a nível de SNC (controladores respiratórios), pulmonares locais e vias aéreas. Trata-se de uma revisão de literatura, sendo usado os descritores com base DeCS (descritores de assunto em ciências da saúde da Bireme), em inglês e português: "Hormônios Esteroides GonadaisSteroid Hormones", "Respiração- Respiration", "Sistema Respiratório- Respiratory System". Foram consultadas as bases de dados eletrônicas PubMed, Web Of Scienci e SciELO. Os estudos incluídos nesta revisão foram baseados em ensaios clínicos e metanálises, estudos que abordassem bem os efeitos dos hormônios sexuais do aparelho respiratório, e estudos publicados nos últimos 5 anos, com exceção de 1 artigo que foi considerado referência base para estudos desta temática. Os critérios de exclusão foram trabalhos publicados em anais de congresso, resumos de eventos científicos e artigos que não estavam disponíveis na íntegra. Observou-se uma crescente linha de evidências de que os hormônios sexuais têm um importante papel em praticamente todos os processos fisiológicos, inclusive na respiração. Os hormônios estrógeno, progesterona e testosterona podem influenciar a função respiratória em animais e em humanos.
\end{abstract}

PALAVRAS-CHAVE: Hormônios Esteroides Gonadais. Fisiologia. Sistema Respiratório.

Acadêmico do curso de Fisioterapia. Departamento de Fisioterapia. Centro Universitário Maurício de Nassau UNINASSAU/JP. CEP: 58083574, João Pessoa, Paraíba. Brasil. E-mail: weslleysaless8@gmail.com. ORCID ID: https://orcid.org/0000-0002-6553-6266

Doutora em Fisioterapia pelo Programa de Fisioterapia da Universidade Federal do Rio Grande do Norte. Departamento de Pneumopediatria. Docente do Centro Universitário Maurício de Nassau - UNINASSAU/JP. CEP: 58053000 , João Pessoa, Paraíba, Brasil. ORCID ID: https://orcid.org/0000-0002-5928-2431 


\section{INTRODUÇÃO}

\begin{abstract}
A função básica do sistema respiratório é suprir o organismo com oxigênio $\left(\mathrm{O}_{2}\right)$ e dele remover o produto gasoso do metabolismo celular, isto é, o gás carbônico $\left(\mathrm{CO}_{2}\right) .{ }^{1} \mathrm{~A}$ literatura científica defende que a respiração não é regulada por hormônios específicos, mas sofre influência de vários deles, pois estes podem apresentar ação estimulante ou inibitória sobre o sistema respiratório. ${ }^{1,2,3}$ Nos últimos anos, tem havido uma crescente conscientização de que os hormônios sexuais têm um importante papel em praticamente todos os processos fisiológicos, inclusive na respiração. 1,3,4
\end{abstract}

Os hormônios estrógeno, progesterona e testosterona podem influenciar a função respiratória em animais e em humanos. ${ }^{1} \mathrm{Em}$ adição, vários distúrbios respiratórios, tais como a apneia obstrutiva do sono, a Síndrome da Morte Súbita do lactente e a Síndrome de Rett também apresentam claras diferenças sexuais nas suas prevalências, conferindo a importância dos hormônios sexuais no controle da respiração. ${ }^{1,2}$

O efeito dos hormônios sobre o sistema respiratório tem sido estudado a várias

\section{MATERIAL E MÉTODOS}

O estudo é caracterizado como sendo uma revisão de literatura e os descritores foram selecionados com base DeCS (Descritores de Assunto em Ciências da Saúde da Bireme). Foram consultadas as bases de dados eletrônicas PubMed, Web Of Scienci e SciELO, utilizando os seguintes descritores em inglês e português: "Hormônios Esteroides GonadaisSteroid Hormones”, “Respiração- Respiration”, décadas. Entretanto, a influência específica dos hormônios sexuais foi confirmada há cinco décadas, quando foi observado que as respostas ventilatórias diferem entre os gêneros e variam de acordo com a fase do ciclo menstrual. ${ }^{1,4}$

A localização de múltiplos receptores de progesterona, estrógeno, andrógeno, prolactina, hormônio luteinizante e gonadotrofina coriônica humana sugerem que estes hormônios podem agir em vários tecidos, incluindo traqueia, pulmões, cérebro e tronco cerebral. ${ }^{1,5} \mathrm{O}$ conhecimento das interações entre hormônios sexuais e respiração propicia novas perspectivas em terapias e estimula pesquisas no intuito de investigar em como o sistema hipotálamo-hipofisário-gonodal influencia no controle da respiração. ${ }^{5}$

O presente artigo tem o objetivo de revisar evidências do efeito dos principais hormônios sexuais, envolvidos na regulação fisiológica da respiração, assim como sua influência na fisiopatologia de desordens respiratórias, evidenciando sua contribuição à nível de SNC (Controladores Respiratórios), pulmonares locais e vias aéreas. 
considerado referência base para estudos desta temática. Os critérios de exclusão foram trabalhos publicados em anais de congresso,

\section{RESULTADOS E DISCUSSÃO}

Existe consenso na literatura científica de que a respiração sofre influência hormonal, pois estes podem apresentar ação estimulante ou inibitória sobre o sistema respiratório, 1,2,3,10 como observado na Tabela 1. resumos de eventos científicos, e artigos que não estavam disponíveis na íntegra.

Tabela 1: Hormônios e substâncias consideradas estimulantes e inibidoras do sistema respiratório

\begin{tabular}{ll}
\hline \multicolumn{1}{c}{ ESTIMULANTES } & INIBIDORES \\
\hline \hline Hormônio do crescimento (GH) & Somatostatina \\
Fator insulínico de crescimento (IGF-1) & TSH \\
Progesterona & Glicina \\
Testosterona & Neuropeptídeo Y \\
Fator de liberação de corticotrofina & Ácido gamaaminobutílico \\
Tireoxina & \\
Estrógenos & \\
Cortisol & \\
Epinefrina & \\
Leptina & \\
Neuropeptídeo Y & \\
Serotonina \\
Histamina
\end{tabular}

Influência dos hormônios sexuais sobre o controle respiratório

O controle ventilatório é um processo complexo e multifatorial, regulado por mecanismos neurais hierarquicamente organizados, composto por três elementos básicos: os sensores que captam as informações acerca das pressões parciais de
Nos últimos anos, tem havido uma crescente conscientização de que os hormônios sexuais têm um importante papel em praticamente todos os processos fisiológicos, inclusive na respiração. ${ }^{6}$

Fonte: Adaptado de Saaresranta ${ }^{6}$ 
A progesterona é considerada como um potente estimulador do centro respiratório. ${ }^{1,4}$ Esta informação corrobora com pesquisas que demonstram que, durante a gravidez, ocorre um forte aumento da excitabilidade do centro respiratório com uma subsequente diminuição da $\mathrm{pCO}_{2}$ alveolar, sugerindo que a sensação de dispneia referida por algumas mulheres, durante a gravidez, possa estar intimamente relacionada a questões hormonais e não apenas a alterações biomecânicas. ${ }^{2,} 5$

Estudos revelam que a combinação de progesterona e estrógenos é eficaz no tratamento de desordens respiratórias como a Doença Pulmonar Obstrutiva Crônica (DPOC) e a Síndrome da Apneia do Sono (SAS). ${ }^{7}$ Em relação a testosterona, existem poucos relatos a cerca dos efeitos no controle respiratório. Alguns afirmam que a testosterona pode ser considerada como um supressor do drive respiratório hipercápnico durante o sono, justificando a maior prevalência de SAS em homens. $^{7,8}$

Influência da progesterona sobre o sistema respiratório

A progesterona é um potente estimuladordocentrorespiratório. Alocalização de múltiplos receptores de progesterona sugere que este hormônio possa agir em vários tecidos, incluindo traqueia, pulmões, cérebro e tronco cerebral. ${ }^{1,5} \mathrm{O}$ aumento da secreção de progesterona pode explicar a hiperventilação e a diminuição de $\mathrm{CO}_{2}$, durante a gravidez e a fase Ißtea do ciclo menstrual. Estas mudanças cíclicas cessam após a menopausa. 9 Em mulheres pré-menopausa, as respostas ventilatórias são aumentadas durante a fase lútea vs. fase folicular do ciclo menstrual, e são superiores quando comparado aos homens, independentemente da fase do ciclo menstrual. ${ }^{2,4,6}$ Durante o sono, as respostas hipoxêmicas são menores em mulheres do que em homens confirmando mais uma vez as diferenças resultantes do gênero no controle ventilatório. ${ }^{7}$ Com a diminuição dos níveis sérios de progesterona, durante o período da menopausa, as mulheres evoluem com a perda desse fator de proteção e podem apresentar desordens respiratórias como a AOS. 7,8,9

Possivelmente, pelo aumento do drive respiratório em geral, a progesterona também aumenta a função das vias aéreas superiores, podendo haver influência dos hormônios sexuais femininos na atividade eletromiográfica dos músculos das vias aéreas superiores e observaram que a progesterona tem impacto substancial e significativo sobre a atividade do músculo genioglosso. Esta informação sugere que a progesterona pode ser considerada como um fator de proteção para o desenvolvimento da Síndrome da Apneia do Sono. 2,9

Influência dos estrógenos sobre o sistema respiratório

Assim como a progesterona, os estrógenos fazem parte da complexa rede de hormônios ovarianos. Os estrógenos normalmente encontrados na espécie humana são: ${ }_{17}^{\beta}$ estradiol (E2), estrona (E1) e estriol (E3). O estriol, produto metabólico do $17^{\beta}$ estradiol, em condições normais é secretado em pequenas quantidades, aumentando durante a gravidez, quando então é secretado pela placente a partir de precursores fetais. Os estrógenos também podem ser produzidos por conversão periférica em outros tecidos como, por exemplo, o tecido adiposo. O papel do tecido adiposo como fonte de estrona é bem evidente após a menopausa. 9,10

Mulheres com ciclo menstrual regular têm menos episódios de hipopneia, durante a fase lútea, quando os níveis séricos de estrógeno e progesterona estão elevados. Como o estradiol necessita da regulação dos receptores de progesterona, 
seus efeitos sobre o sistema respiratório são semelhantes ao da progesterona. 4,7 Já no período damenopausa, mulheresquefazemuso de terapia de reposição hormonal apresentam menos episódios de obstrução brônquica, embora existam evidências de que o estrógeno pode deteriorar a respiração de pacientes com asma e estas necessitarem fazer uso de uma quantidade maior de broncodilatadores. ${ }^{10}$

Nesse contexto, alguns estudos demonstram associação positiva entre terapia de reposição de estrógeno e sintomas asmáticos. O broncoespasmo induzido por estrógeno em mulheres pós-menopausa com obstrução severa de vias aéreas está bem relacionado com o uso de contraceptivos orais. $^{8,9}$

Influência da testosterona sobre o sistema respiratório

\section{CONSIDERAÇÕES FINAIS}

Identificou-se uma grande relação dos hormônios sexuais nos processos fisiológicos, inclusive na respiração. Sendo destaque os hormônios estrógeno, progesterona e testosterona que podem intervir diretamente na função do sistema respiratório, tanto em animais quanto em humanos. Mais estudos sobre a relação dos hormônios sexuais e a
Embora a testosterona seja um hormônio sexual masculino, ela pode ser encontrada, em baixas concentrações, em mulheres. Apesar de ser o único andrógeno que tem sido atribuído ao controle respiratório o seu papel ainda não está bem esclarecido. ${ }^{11}$

Evidênciasapontamquea testosterona aumenta a resistência ventilatória hipóxica e a taxa metabólica aumentando o risco da AOS. ${ }^{9,10}$ Em homens com doença respiratória crônica as concentrações séricas de testosterona correlacionam-se positivamente com a PaO2. ${ }^{10,11}$

Autores defendem que anormalidades endócrinas são revertidas três meses de terapia com pressão positiva contínua em vias aéreas (CPAP). Contudo, níveis de testosterona têm sido observados em pacientes com AOS sem qualquer efeito a partir do tratamento de CPAP. ${ }^{11}$ respiração devem ser estimulados, assim como a relação desses hormônios com os demais sistemas. Estes novos estudos podem diretamente intervir nas terapias usadas nos pacientes, proporcionando sempre a melhor conduta terapêutica, com as particularidades para cada indivíduo.

\section{INFLUENCE OF SEX HORMONES ON PHYSIOLOGICAL REGULATION OF BREATHING: A LITERATURE REVIEW}

\section{ABSTRACT}

The respiratory system is responsible for oxygenating all blood that is low in oxygen (O2) and removing our blood or carbon dioxide ( $\left.\mathrm{CO}_{2}\right)$. Even if there is no use of sex hormones related to the breathing 
process, the literature shows that there is an influence, especially when they may have stimulating or inhibiting action on the respiratory system. This study aimed to review the literature on the effects of the primary sex hormones affected by the physiology of breathing, as well as their influence on the pathophysiology of respiratory diseases, highlighting their contribution to the level of the CNS (respiratory controllers), local lungs and airways. The study is selected as a literature review, and this search was conducted in the second half of 2019. Descriptors were selected based on DeCS (Bireme health science subject descriptors). They were consulted as electronic databases PubMed, Web of Science, and SciELO, using the following descriptors in English and Portuguese: "Gonadal Steroid Hormones - Steroid Hormones," "Breathing-Breathing," "Respiratory System-Respiratory System." The studies included in this review were conducted in clinical trials and meta-analyses, studies that address well the effects of respiratory hormones, and studies published in the last five years, except for one article that was considered based on studies on this subject. Exclusion criteria were congress proceedings, abstracts of scientific events, and articles that were not fully available. Observe a growing line of sex hormones that play an essential role in virtually all physiological processes, including breathing. The hormones estrogen, progesterone, and testosterone may influence respiratory function in animals and humans.

KEYWORDS: Gonadal Steroid Hormones. Physiology. Respiratory System.

\section{REFERÊNCIAS}

1- Al-Shamkhi N, Alving K, Dahlen SE, Hedlin G, Middelveld R, Bjerg A, Ekerljung L, Olin AC, Sommar J, Forsberg B, Janson C, Malinovschi A. Important non-disease-related determinants of exhaled nitric oxide levels in mild asthma - results from the Swedish GA(2) LEN study. Clin Exp Allergy. 2016 Sep; 46(9):1185-93.

2- Boukari R, Laouafa S, Ribon-Demars A, Bairam A, Joseph V. Ovarian steroids act as respiratory stimulant and antioxidant against the causes and consequences of sleep-apnea in women. Respir Physiol Neurobiol. 2017 May; 239:46-54.

3- Chen HH, Lu J, Guan YF, Li SJ, Hu TT, Xie ZS, Wang F, Peng XH, Liu X, Xu X, Zhao FP, Yu BL, Li XP. Estrogen/ERR-a signaling axis is associated with fiber-type conversion of upper airway muscles in patients with obstructive sleep apnea hypopnea syndrome. Sci Rep. 2016 Jun; 6:27088.

4- Frump AL, Selej $M$, Wood JA, Albrecht $M$, Yakubov B, Petrache I, Lahm T. Hypoxia Upregulates Estrogen Receptor $\beta$ in Pulmonary Artery
Endothelial Cells in a HIF-1a-Dependent Manner. Am J Respir Cell Mol Biol. 2018 Jul; 59(1):114-26.

5- Wang X, Pan L, Zou Z, Wang D, Lu Y, Dong Z, Zhu L. Hypoxia reduces testosterone synthesis in mouse Leydig cells by inhibiting NRF1-activated StAR expression. Oncotarget. 2017 Mar; 8 (10):16401-13.

6- Saaresranta T, Polo O.Hormones and breathing. Chest. 2002 Dec; 122(6):2165-82.

7- Levental S, Picard E, Mimouni F, Joseph L, Samuel TY, Bromiker R, Mandel D, Arish N, Goldberg S. Sex-linked difference in blood oxygen saturation. Clin Respir J. 2018 May; 12(5):1900-04.

8- Molina FD, Suman M, Carvalho TBO, Piatto SRT; Maniglia JV, Tognola, WA. Avaliação dos níveis séricos de testosterona em pacientes com síndrome da apneia obstrutiva do sono. Braz. J. Otorhinolaryngol. vol.77 no.1 São Paulo Jan./Feb. 2011. 
revista de NOVA
ciêncios NOSP

da saû́des ESPERANÇA

9- Peixoto C, Grande AJ, Mallmann MB, Nardi AE, Cardoso A, Veras AB. The effects of dehydroepiandrosterone on sexual function: a systematic review. CNS Neurol Disord Drug Targets. 2018; 17 (9):706-11.

10- Grimm A, Biliouris EE, Lang UE, Götz J, Mensah-Nyagan AG, Eckert A. Cell Mol Life Sci. Sex hormone-related neurosteroids differentially
VOLUME 17 - NÚMERO 3 - DEZ/2019 ISSN ELETRÔNICO 2317-7160

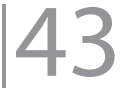

rescue bioenergetic deficits induced by amyloid- $\beta$ or hyperphosphorylated tau protein. 2016 Jan; 73(1):201-15.

11- Yu RM, Chaturvedi G, Tong SK, Nusrin S, Giesy JP, Wu RS, Kong RY. Evidence for microRNA-mediated regulation of steroidogenesis by hypoxia. Environ Sci Technol. 2015 Jan; 49 (2):1138-47. Environ Sci Technol. 2015 Jan; 49 (2):1138-47. 